Diese Publikation wurde zuerst veröffentlicht in/this publication has been published first in:

Lange, Bastian; Ehrlich, Kornelia (2008): Geographien der Szenen. Begriffserklärungen und zwei Fallvergleiche im Feld der urbanen Kultur- und Kreativwirtschaft von Berlin und Leipzig, in: Sociologia Internationalis: internationale Zeitschrift für Soziologie, Kommunikations- und Kulturforschung, Band 46, Heft 2, Berlin: Duncker \& Humboldt, S. 237-261. 


\title{
GEOGRAPHIEN DER SZENEN - BEGRIFFSKLÄRUNGEN UND ZWEI FALLVERGLEICHE IM FELD DER URBANEN KULTUR- UND KREATIVWIRTSCHAFT VON BERLIN UND LEIPZIG
}

\author{
Von Bastian Lange und Kornelia Ehrlich
}

\section{Einleitung}

Der Beitrag präzisiert eine begriffliche Unschärfe in der Sozial-, Kultur- und Wirtschaftsgeographie. Die Autoren argumentieren, dass der Begriff Szene in der Sozial-, Kultur- und Wirtschaftsgeographie defizitär konzeptionalisiert wird, obwohl er in verschiedenen Bereichen des Alltags, der angewandten sowie der konzeptionellen Wissenschaft verstärkt breite Verwendung findet: Einerseits wird Szene umgangssprachlich zur Beschreibung und Verortung kreativer Gruppierungen, flüchtiger Vergemeinschaftungen aber auch professioneller Netzwerke in der sogenannten Kreativ- und Wissensökonomie angewandt. Andererseits haben kultur- und sozialwissenschaftliche Disziplinen in jüngster Zeit konzeptionelle Ansätze entwickelt, um mit Hilfe des Konzepts Szene eine Beschreibung der Funktionalität des Städtischen zu erreichen. Die Leitfrage des Beitrags lautet daher: Kann der Begriff Szene als ein kultur- und wirtschaftsgeographisches Konzept verstanden werden? Was leistet dann ein Konzept „Szene“?

\section{Aufbau}

Der Beitrag ist in vier Teile gegliedert. In einem ersten wird gezeigt, welche „Verbreitung“ die Verwendung des Begriffs Szene aufweist. Notwendigerweise erfordert dies eine systematischere Darstellung, die im zweiten Teil anhand einer disziplinären Verortung des Begriffs Szene erfolgt. Sie wird im Wesentlichen von der Annahme getragen, dass die Alltagswelt der Nachmoderne von tiefgreifenden sozialen Transformationsprozessen begleitet wird: Prozesse der Individualisierung aber auch der Emergenz von neuen Vergemeinschaftungen, Veränderungen von institutionellen Arrangements sowie eine generelle kulturelle sowie ökonomische Pluralisierungen durchziehen nachmoderne Gesellschaften zu Beginn des 21. Jh. Es bilden sich segmentierte eigenlogische Lebenswel- 
ten ab/heraus. Dabei haben postmodernistische Sozialtheorien neue soziale Verunsicherungen sowie instabile soziale Verankerungen in den Bereichen Arbeit und Leben diagnostiziert, die wiederum ein Neuverhandeln von sozialen Grenzziehungen zwischen Individuum und Gesellschaft mit sich bringen. Die Etablierung von Vergemeinschaftungen, die alltagssprachlich mit dem Begriff Szene angesprochen werden sind Ausdruck neuer Formen von sozialer Kontinuität bzw. Kollektivität. Der zweite Teil stellt die disziplinäre Verwendung und Konzeptionalisierung von Szene dar. Im dritten Teil werden die eingangs erläuterten konzeptuellen Überlegungen exemplarisch an zwei urbanen Szenerien erläutert. Die dabei vorgestellten Beispiele sind das Ergebnis zweier qualitativer Fallrekonstruktionen in Rahmen von empirischen Erhebungen. Im Vordergrund dieser Darstellung steht die Frage, inwiefern sich Szenen nicht nur als Ausdruck neuer Interaktionsbeziehungen in Wirtschaftsbereichen - genauer in Teilmärkten der Kultur- und Kreativwirtschaft $^{1}$ - und somit semi-professionellen Arbeitsbeziehungen verorten lassen, sondern vielmehr darüber hinaus in der Lage sind als analytisches Konzept Konstituierungen und Konstituierungslogiken des Städtischen zu erbringen.

Der vierte Teil schließlich macht deutlich, dass Szenen einen Ausdruck spezifisch urbaner Prozesse der Verräumlichung sozialer Vergemeischaftungen darstellt. Szenen, so argumentieren die Autoren, leisten ein Verständnis zur Eigenlogik des Städtischen und geben somit Hinweise, wie sich Städte unterscheiden. In dieser Folge leistet Szene als analytisches Konzept Hinweise zur Formierung des Städtischen. Dadurch zeigt sich anhand des hier eingeführten Verständnisses von Szene, wie soziale Vergemeinschaftungen ihren Niederschlag sowie eine ReMaterialisierung im Städtischen finden. Das bedeutet weniger, dass sich Szenen ausschließlich über ihre Verräumlichung verorten lassen, sondern vor allem, dass sich Szenen über Prozesse der Raumaneignung formieren und diese Szenedynamiken wiederum in der Lage sind, Räume herzustellen.

\footnotetext{
1 Nach Definition des Bundesministeriums für Wirtschaft und Technologie ist der verbindende Kern jeder kultur- und kreativwirtschaftlichen Aktivität ein schöpferischer Akt (BMWi (2009): Gesamtwirtschaftliche Perspektiven der Kultur- und Kreativwirtschaft in Deutschland. Berlin: Bundesministerium für Wirtschaft.). Dazu zählen beispielsweise literarische, musische, gestalterische und architektonische Inhalte und Werke. Diese Produktionsprozesse können in Unikate, Liveaufführungen, serielle Produktionen oder Dienstleistungen münden. Neben kleinteiligen Kulturunternehmern zählen aber auch die Unternehmen der Werbewirtschaft, der Designwirtschaft, der Filmwirtschaft und der Software-/ Games-Industrie zum heterogenen Branchenkonglomerat „Kultur- und Kreativwirtschaft".
} 


\section{Szene - eine (unvollständige) Landkarte der Verwendung des Begriffs Szene}

Der Begriff Szene wurde bisher im wirtschaftsgeographischen Theorie- sowie Anwendungskontext nicht eindeutig verwendet und ist somit erklärungsbedürftig. Eine exponentielle Zunahme des Begriffs Szene ist im fachwissenschaftlichen (1), fachadministrativen (2) aber auch alltagssprachlichen Umgang (3) zu finden:

(1) Das Vorhandensein von Szenen und kreativen Milieus wird für die Herausbildung attraktiver Wirtschaftsstandorte als relevant und unabdingbar angesprochen (Hospers 2003, 143 ff.). Dies leitet sich aus der Beobachtung ab, dass neue Medien und Kulturproduzenten spezifische Raumansprüche haben und diese an bestimmten Orten vorfinden. Das Vorhandensein von engmaschigen interaktionsreichen Produktionsfeldern in urbanen Kontexten gilt heute als zentrales Kennzeichen von Metropolen (Hesse/Lange 2007, 65). Unternehmerische Akteure der Kultur- und Kreativwirtschaft sind im Wesentlichen darauf angewiesen, an der Herstellung dieser Räume beteiligt zu sein. Für den Wandel hin zu einer „kreativen Stadt“ sind laut Reckwitz „Kunstszenen“ verantwortlich, die aufgrund ihres performativen Charakters in der Lage sind, den Prozess der Selbstkulturalisierung einer Stadt ganz wesentlich anzutreiben (Reckwitz 2009, 22-23)

Diese neue Art von neuer symbolischer Ökonomie hat nicht nur eine Affinität zu sozio-kulturellen Milieus einer Metropole. Sie siedelt sich auch innerhalb der Großstadt in ganz bestimmten Räumen an. Prototypisch hierfür sind codierte innerstädtische Szene-Distrikte, gentrifizierte und ästhetisierte Altbauquartiere mit einem hohen Lifestylefaktor (so wie Kaffeebars mit Wireless-Lan-Netzwerken), aber auch umgebaute Hafen- oder Gewerbestandorte mit Wohnlofts und Ateliers, in denen die Vorstellung von raumgreifenden Tätigkeiten sprichwörtlich ihren Platz finden kann. Preiswerte Mieten haben hier ihren besonderen Wert und machen Städte wie insbesondere Berlin aber auch Leipzig attraktiv gerade auch für eine Klientel, das oft unter prekären Randbedingungen arbeitet und sich übliche Großstadtmieten kaum leisten kann.

(2) In jüngster Zeit hat der Begriff Szene auch Eingang in fachadministrative Studien gefunden, um einerseits Marktformierungen sowie Akteursnetze gerade in kreativ- und wissensökonomischen Bereichen adäquater zu beschreiben (Weckerle et al. 2007, 108 ff., SMWA, 2008, 78 ff.). Der Begriff Szene wurde dabei auf das Netzwerkverhalten von neuen professionellen Marktteilnehmern in der Kultur- und Kreativwirtschaft angewandt. Szenen beschreiben dabei Formen des Austauschs zwischen jungen sowie etablierten Marktakteuren. Das Vorhandensein von als kreativ bewerteten Szenen hebt auf ein bestimmtes Maß 
an kommunikativer und interaktiver Dichte, ein Maß an „kritischer Masse“ ab. Neben den Marktteilnehmern innerhalb der Teilmärkte weisen auch Vertreter etablierter Märkte sowie Fachadministrationen auf die zunehmende Relevanz der „kreativen Szenen“ für das wirtschaftliche Gefüge einer Stadt hin, da in diesen informellen Netzwerken Innovationsprozesse vollzogen werden, von denen gerade andere Branchen profitieren. Sogenannte „Unternehmerische Szenen“ (SMWA, 2008, 78 ff.). in Form von innovativen und kreativen Interaktionsbeziehungen zwischen Marktteilnehmern, scheinen in diesem Zusammenhang eine Inkubatorenfunktion für regionale Wirtschaftsprozesse zu haben. Das Vorhandensein dieser unternehmerischen Marktteilnehmer wäre danach entscheidend für die regionale Wettbewerbsfähigkeit.

(3) Der Begriff Szene hat darüber hinaus in der alltagssprachlichen Verwendung eine weitere Verbreitung durchlaufen. Im Wesentlichen wird dieser in Print- und Onlinemedien zur Beschreibung von Kulturereignissen und erregungsreichen Orten herangezogen. Der Begriff Szene hat dabei die Funktion politische, kulturelle oder lebensweltliche Mikrokollektive (z. B. rechte oder linke Szene, Jazz-Szene, oder HackerSzene), die sich aufgrund ihrer thematischen Fokussierung abseits von gesellschaftlichen Mehrheiten bewegen, sprachlich zu bündeln. Der Begriff wird umgangssprachlich auf Phänomene wie „Alternativszene“, aber auch Szenetreffpunkt, Szeneviertel, Künstlerszene und Kreativszene angewandt. Ebenso findet der Begriff eine ausufernde Verwendung zur Beschreibung von kulturellen Events in Stadt- und Kulturmagazinen.

\section{Disziplinäre Verwendungen des Begriffs Szene}

\section{Soziologisch-handlungstheoretisches Verständnis von Szene}

Einen wesentlichen Beitrag zum Verständnis von Szenen leistet die Jugendsoziologie. Grundsätzlich bedeutet Szene für Ronald Hitzler ein Gesellungsgebilde, das nicht aus gemeinsamen Lebenslagen oder Standesinteressen der daran Teilhabenden entsteht, sondern aus Interesse an einem thematischen Kern (Hitzler et al. 2001, 5-10). Als Bedingung der Teilhabe weisen Szenen einen signifikant geringen Verbindlichkeitsgrad und Verpflichtungscharakter auf. Von Subkulturen unterscheiden sich Szenen wesentlich durch ihre Diffusität im Hinblick auf Inklusion und Exklusion. Von Cliquen differenzieren sie sich wesentlich durch deutlich geringere Altershomogenität, durch geringere Interaktionsdichte und durch geringe Orts- und Sozialraumbindung, d. h. durch Translokalität. 
Szenen stellen für juvenile Menschen komplexe soziale Allianzen für Leidenschaften, Interessen, Neigungen und Vorlieben dar, die sie in ihrem alltäglichen sozialen Interaktionsraum nicht erfüllt und verwirklicht sehen. Szenen bieten die Option, Gleichgesinnte zu finden und entwickeln dabei eine eigene Kultur (ebd.). Eine Szene lässt sich als eine Form der Vergemeinschaftung von Akteuren charakterisieren, die bestimmte materiale und mentale Formen der kollektiven Selbst-Stilisierung teilen, um diese Teilhabe wissen und diese Gemeinsamkeiten kommunikativ stabilisieren, modifizieren oder transformieren.

Dem voluntaristischen Selbstorganisationspotenzial von Szenen widersprechend, weisen Hitzler / Bucher / Niederbacher darauf hin, dass sich Szenen um einerseits physische Orte, andererseits um eine funktionale Organisationselite anordnen (Hitzler et al. 2001, 27). Gleichwohl konzipiert Ronald Hitzler Orte als ein Gegenpol zum Sozialen. Damit konstruiert er eine Dualität zwischen Sozialem gegenüber Raum. Physisch-räumliche Treffpunkte geben aber gerade den labilen sozialen Bindung, die zwischen Szenegängern vorherrschen, ein temporär stabilisierendes Identifikations- und Kommunikationsangebot. Funktionale Organisationseliten strukturieren $\mathrm{Ab}$ - und Begrenzungen innerhalb der Szenetreffpunkte sowie die Durchlässigkeit zu anderen Szenen.

\section{Wirtschaftssoziologisches Verständnis von Szene}

Auffallend breite Rezeption findet der Begriff Szene in jüngster Zeit im Bereich der Beschreibung von Marktgeschehen - insbesondere durch junge Marktteilnehmer - in der Kultur- und Kreativwirtschaft (Weckerle et al. 2007, 108, Lange 2007, Lange / Mieg 2008). Ebenso werden aus wirtschaftssoziologischer Perspektive beispielsweise das Gründungsgeschehen in jungen Märkten als im Wesentlichen szene-basiert beschrieben (Schallberger 2003). Junge Märkte in der Kultur- und Kreativwirtschaft sind vielfach durch Instabilitäten sowie ein Maß an Unordnung und Vielschichtigkeit gekennzeichnet (Rossiter 2007). Formalisierte Konzepte und statische Verständnisse von Akteuren sowie deren Beziehungen untereinander geraten somit schnell ins Wanken. Harrison White betont daher die Übergangsbereiche zwischen sozialen Netzwerken, sozialen Räumen und klaren, rigiden Identitäts- und Rollenkonzeptionen in sozialen Netzwerken und wendet sich gegen klar abgrenzbare Begriffe und formal-technische Netzwerkformationen (White 2002). Aus dieser Perspektive interpretiert Gernot Grabher Whites' Verständnis von Akteur und Person als „nicht notwendigerweise souveränen Gestalter von Netzwerkbeziehungen, sondern selbst als Knoten kondensierter Geschichten und Identitäten, die sich an den Schnittstellen multipler Netzwerke sedimentieren"(Grabherr 2006, 104). 
Die intellektuell herausfordernde Perspektive von White, die Brüchigkeit, Fuzziness und Transgression als systemimmanente Elemente von Kollektiven, Netzwerken und Märkten anspricht, kann zunächst als ein Gegenentwurf zu einem rigiden und hermetischen Verständnis von z. B. formalen Netzwerkkonzepten verstanden werden. Gerade in der Kulturund Kreativwirtschaft zeigen sich diese neuen Arbeits- und Organisationsformen in Gestalt von "disorganized labour“ (Rossiter 2007, 156 161), welche einen prinzipiell wechselhaften und brüchigen Charakter zum Ausdruck bringen. Ned Rossiter weist darauf hin, Netzwerksozialitäten konsequenterweise immer wieder neu organisiert werden müssen.

Auch in urbanen Kontexten geben sich auffallende Passungen zwischen dem grundsätzlichen Netzwerkverständnis von Harrison White und jüngeren Formen der Konstituierung von Sozialräumen zu erkennen. Insbesondere Kleinstunternehmer aus den der Kultur- und Kreativwirtschaft leisten dabei wichtige Impulse (Ertel 2006, 18-20). Sie lassen sich als Repräsentation „kultureller Individualisierung“ (McRobbie 2005, 21) ansehen und artikulieren eine neue Subjektivierung von Arbeit, die sich verstärkt in sozialen Netzwerken vollzieht: Hochgradig flexibilisierte Arbeit entgrenzt sich zugleich in gemischte privat-familiäre Sozialräume, die nur durch eben diese Unterstützungsstrukturen getragen und bewältigt werden. Wesentliche Kompetenzen der Bewältigung dieser professionellen Anforderungen wurden meistens frühzeitig „nebenher“ im Freizeitbereich erworben. Nicht-kommerzielle Clubs, Sport- und Spielveranstaltungen auf Brachflächen oder leer stehenden Gewerberäumen funktionieren aufgrund der subkulturellen Kompetenz, der Lust am Experiment sowie einer nur dort möglichen Atmosphäre des Ausprobierens. Zum Künstler des räumlichen Experiments hat sich aber eine unternehmerische Praxis angelehnt, denn nur mit dieser Verbindung konnte das Leben in Freizeit- und Clubszenen nachhaltig finanziert werden. Szene- und Ortskompetenzen sind somit informelle, aber zentrale Qualitäten für den Einstieg in Marktkontexte, in denen (neue) Erwerbsbiographien entwickelt werden können. Clubsozialisationen sind Dreh- und Angelpunkte, von denen aus der Zugang zu Arbeit neu verhandelt sowie die Realisierung von neuen Projekten sowie unternehmerischen Prozessen entwickelt werden kann.

So genannte Unternehmerische Szenen leisten für die Marktteilnehmer eine wichtige Aufgabe: In diesen informellen Netzwerken werden neue Trends (Stile, Moden etc.) und symbolische Innovationen sprachlich verhandelt. Gerade symbolische Produkte (z. B. Zeichen, Stile, Bilder etc.) der Kultur- und Kreativwirtschaft bedürfen einer ersten Bewertung von Kreativexperten, um überhaupt als relevantes marktfähiges „Produkt“ wahrgenommen zu werden. Diese informellen Bewertungen finden in unternehmerischen Szenen statt. Aus den Szenen können sta- 
bilere Strukturen wie marktförmige Vernetzungsplattformen, Messen oder dergleichen erwachsen. Szenen organisieren sich einerseits um physische Orte, andererseits um eine funktionale, aber nicht über Autorität verfügende Organisationselite.

Existieren kritische Massen und zumindest temporär dichte Kommunikationsnetze derartiger unternehmerischer Szenen prägt das Räume: Stadtteile werden mit dem Vorsatz „in“- oder Szeneviertel bewertet. d. h. dort herrscht ein anregungsreicher und stimulierender kultur- und kreativwirtschaftlicher Rahmen vor. Derartige unternehmerische Szenen wird eine Inkubatorfunktion für regionale Wirtschaftsprozesse zugewiesen. Unternehmerische Szenen sind darüber hinaus interessant, weil sie ein hohes Maß an Selbstorganisation in sich tragen: Sie schaffen sich notwendige Strukturen wie Messen, Absatzmöglichkeiten und Ladengeschäfte selbst. Die Szene-Akteure versprechen sich von diesen informellen Netzwerken Zugang zu Informationen, die sie über formalisierte Kommunikationswege (z. B. Branchenblätter etc.) nicht erhalten würden. Eine unternehmerische Szene lässt sich also als eine Form der sozialen Vergemeinschaftung von unternehmerischen Akteuren charakterisieren, wie dies in zahlreichen Teilmärkten der Kultur- und Kreativwirtschaft der Fall ist (z. B. im Bereich des Musikmarktes die Independentszene, oder im Architekturmarkt die Architektenszene etc.). Szenenkontexte lösen damit einen dringend benötigten Bedarf nach Kommunikation und Austausch ein.

\section{Sozialphänomenologisches Verständnis von Szene}

Sozialphänomenologische Verständnisse werden hier als Gegenentwurf zu den vorangegangenen handlungstheorieorientierten Ansätzen eingeführt. Sie sprechen Szenen aufgrund ihres temporären Charakters als hochdynamische Konstrukte an. Städte werden mit Hilfe von inszenierten Erlebniswelten als eine Bühne für performative Praktiken zur Attraktivitätssteigerung des Städtischen errichtet (Janson 2004, 20-21). Ähnlich wie Alban Janson diagnostiziert Jürgen Hasse, dass Stadträume zunehmend auf „das subjektive Erleben“ ausgerichtet sind und dadurch der Fokus auf die „eigene Situation“ gerichtet wird (Hasse 2002, 19-25). Situative Qualitäten nehmen in Städten einen höheren Stellenwert ein als eine rein funktional ausgerichtete Raumorganisation. Fragen des „menschlichen Erlebens" haben den Charakter des „szenischen Erlebens, in dem wir Akteure und Zuschauer zugleich sind" (Hasse 2002, 21; Hasse 2008).

In ähnlicher Art und Weise hat Alan Blum vor einem sozialphänomenologischen Hintergrund eine Theorie der Szene zur Beschreibung von 
Stadträumen entwickelt (Blum 2001; Blum 2003). Aus dem Verständnis der „Stadt als Bühne“ und einer urbanen Theatralität erkennt Blum urbane Anordnungen im Sinne eines sich selbst Erlebens, Sehens, Spürens und zugleich Gesehenwerdens. Ein existenzielles „Sich Entziehen“ dieser Situationen und Anordnungen ist nicht möglich.

Auch als (orts-)fremder und mit den spezifischen urbanen Situationen nicht vertrauter Mensch muss man sich zu bestimmten Situationen positionieren und in diesem Sinn performativ agieren. Alle performativen Aktionen unterliegen einer Grammatik: einer Grammatik des Szenischen und der Szenen (Blum 2001, 10). Szenen werden durch Blum in diesem Sinn sozialphänomenologisch anhand von Kriterien wie Regelmäßigkeit, Reichhaltigkeit, Vergänglichkeit, Vergemeinschaftung, Performanz, Transgression, Schauspiel sowie dem Verhältnis Öffentlichkeit vs. Privatheit analysiert (Blum 2001, 10-30): Alan Blum fügt zudem die „Creative City“ hinzu, die er als „relentless circulation of capital in search for markets" anspricht, die sich über die Zwänge des Lokalen hinwegsetzt (Bum 2001, 25). Blum zufolge werden Szenen aus marktwirtschaftlicher Sicht zwecks Profitmaximierung bewusst kalkuliert, konstruiert und entworfen. Szene könnte daher auch als ein ökonomisches Modell verstanden werden, das aber nicht dem originären Konzept der Zielgruppe entspricht. Blum versteht Szene dagegen als ein dialektisches Phänomen: Ihre Vitalität kann zu einer Ware werden, wiewohl die unternehmerische und korporative Absorbierung der Szene in kapitalistische Verwertungsprozesse ein integraler Bestandteil ihrer sozialen Mobilisierungskräfte sowie ihrer Anziehungskraft ist (Blum 2001, 26).

Der Bezug zum Kriterium der kreativen Stadt liegt - Blum zufolge darin, dass Szenen in ihrem Wesenskern das Versprechen in sich tragen, durch die Teilnahme an Szenepraktiken zum Bestandteil der Bohèmien'schen Aktivitäten der Stadt zu werden. Diese Kernpraktiken diffundieren im Verlauf der Zeit zu mundanen Praktiken, in deren Folge der ästhetische, originäre und spielerische Charakter von Szenen sukzessive vermindert wird. Verallgemeinern und lösen sich Szenepraktiken in breitere gesellschaftliche Segmente auf, so transformieren sich diese und diffundieren unter Umständen zu massenkompatiblen Phänomen. Oder sie werden mythologisiert, d. h. ihres ursprünglichen authentischen Erfahrungsraums beraubt.

Städtische Kreativität basiert somit im Verständnis von Blum auf Szenen und in diesem Sinn sozialen Vergemeinschaftungen, die als Generator permanent neue Kultur-, Deutungs- und Sinnmuster, Identifikationsangebote und stilbildende Verortungen hervorbringen. Die Vergänglichkeit von Szenen erlaubt ihren Mitgliedern, sich neu zu erfinden und sich so kulturell über einen Zeitverlauf zu transformieren (Blum 
2003, 183). Szenegänger wählen Orte aus, der Prozess der Vergemeinschaftung aber ist an deren Erfindung und Codierung gekoppelt.

\section{Subkulturverständnis Szene}

Der Begriff der Subkultur ist seit seinem Aufkommen in den 1940erJahren einem stetigen Bedeutungswandel unterworfen. Vor allem das 1964 an der Universität Birmingham gegründete Centre for Contemporary Cultural Studies (CCCS) beeinflusste über 20 Jahre maßgeblich Themen und Methoden subkultureller Analysen (Gelder / Thornton 1997, 83). Die Forschungen am CCCS befassen sich vor allem mit den proletarischer Subkulturen der 1960er- bis frühen 1980er-Jahre.

Das am CCCS konzipierte Verständnis von Subkultur geht davon aus, dass der soziale Referenzpunkt für Subkulturen die Klasse ist, wodurch der subkulturelle Status von Jugend-Subkulturen mit der Klassenunterordnung der Arbeiterklasse zusammenhängt, aus der die Jugend-Subkulturen jeweils hervorgehen (Gelder 1997, 84). Des Weiteren ist der ausgebildete Stil innerhalb der Subkulturen von zentraler Bedeutung, spielt die Transformation kultureller Objekte der dominanten Kultur sowie das sich Aneignen dieser Objekte eine wichtige Rolle für die Ausbildung und Territorialisierung von Subkulturen.

Als direkte Kritik auf die Konzeption von Subkultur am CCCS entwarf Stanley Cohen ein alternatives Analyse-Konzept (Cohen 1997): er plädiert für eine ethnographische Analyse von Subkulturen, um die inneren Mechanismen möglichst ungefiltert beobachten und beschreiben zu können; weiterhin fordert er Subkulturen generell weniger in Bezug auf die Kategorien Spektakel bzw. Widerstand zu untersuchen, sondern sie vielmehr als Reaktions- und Verarbeitungsmechanismen Jugendlicher zu begreifen, die dadurch versuchen mit aktuellen Problemen und Transformationsprozessen umzugehen (Gelder 1997, 146).

\section{Szene in den postmodernistischen Ansätzen}

Jüngere Ansätze, die sich auf Konzepte von Performativität/Performanz beziehen, begreifen die Stadt als situativen Topos - als Ausdruck eines situativen Urbanismus (Legnaro 2004; Lange 2008, 160). Sie fragen nach den aktuellen Formen städtischer Wahrnehmung und nach Aneignungen städtischer Räume. Stadt ist nicht mehr ganzheitlich-funktional wahrnehmbar, sondern wird vielmehr als rhizomatisches Geflecht im Prozess des Gehens, im Ver-Laufen erfahrbar sowie in Verbindung mit jüngeren medialen Formen (Pop, Film, Public Art...) (Berking/Löw 2005, 10-11; Klein 2005a, 25). „Stadt“ wird als bestimmte Form der Ur- 
banität und des Lokalen verstanden. Pop, Film und Public Art sind Praxisformen, die durch ihre Reflexion der Materialität des Städtischen ein neues Wechselverhältnis zeigen. Sie sind in der Lage neue Topoi des Städtischen zu formulieren, sie ermöglichen ästhetische Erfahrungen des Städtischen und produzieren z. B. über Klangwelten neue Formen lokaler Urbanität (de Certeau 1988). Jede Stadt hat demnach ihren Song!

Die dabei angelegten Metaphoriken stellen Formen der Wirklichkeitsproduktion dar. Neue Formen lokaler Urbanität sind somit als performative Aneignungsvorgänge innerhalb von offenen Teilsystemen anzusprechen. Performative Prozesse der Aneignung sind hochgradig situativ: sie artikulieren sich am offensichtlichsten an den Sollbruchstellen (Brachflächen, Baustellen, Leerstand, temporären Nutzungen) des urbanen Stadtkörpers und seiner Teilflächen, die oftmals außerhalb der Verwertung sind und brach liegen. Die performativen Praktiken der temporären Neuinwertsetzungen (durch Kunst-, Sport-, Tanz-, Feierveranstaltungen) sind meistens nicht-linear, disharmonisch-dissonant und nicht repräsentierend $\mathrm{zu}$ anderen etablierten Praxisformen aufzufassen (Klein 2005a). Die theatrale Praxis der Szenen konfiguriert und misst neue Räume aus. Wesentliche Basis dieser Praxis ist dabei die Erkenntnis, dass das, was gemacht wird, immer auch dargestellt wird. Dies gilt im Besonderen für die Formierung des öffentlichen Raums, auf den zahlreiche Akteure einzuwirken versuchen. Die Theatralisierung des Sozialen, die Szenifizierung des urbanen Raums, die Musealisierung des öffentlichen Raums - alle arbeiten in unterschiedlicher Art und Weise daran die Akkumulation von symbolischem Kapital zu forcieren. Während die Stadt als Marke einheitlich und mit einem klaren Image und einer Botschaft versehen sein muss, ist die rhizomatische Stadt in postmodernistischen Verständnisse vielschichtig, fragmentarisch und in ständiger Veränderung (Lange 2008, 161-162).

\section{Eine kontextspezifische Szenedefinition - Unternehmerische Szenen in der Kultur- und Kreativwirtschaft}

Im Folgenden werden - auf der Grundlage dem in Kapitel 2 ausgeführten disziplinären Verständnis von Szene - Parameter benannt, die in eine kontextspezifische Definition von Szene münden. Verbunden wird diese Darstellung mit Elementen zweier unternehmerischer Szenen in zwei unterschiedlichen Kontexten - Berlin und Leipzig -, um darüber hinaus Aufschlüsse über ihre spezifische Artikulation zu erhalten. Zum besseren Verständnis der jeweiligen Situationen erfolgt eingangs eine knappe Beschreibung des Kontextes, der - so wird im Folgenden gezeigt - im wesentlichen durch unternehmerischen Szenen der Kultur- und Kreativwirtschaft artikuliert wird. 


\section{Methodische Hinweise}

Fallrekonstruktive Methoden tragen dem hier vorliegenden jungen Forschungsgegenstand Rechnung, da sie eine große thematische und inhaltliche Offenheit an den Tag legen (Bogner 2002a, 2002b; Froschauer 2000). Ausgangspunkt der folgenden Analyse ist daher die Annahme, dass das unternehmerische Handeln innerhalb der Kultur- und Kreativwirtschaft sowohl in sozialstrukturelle als auch in sozialräumliche Kontexte wiederum eingebettet werden muss. Eine empirische Erhebungsmethode muss in der Lage sein, die Frage nach der Aneignung von Orten zu beantworten sowie die Frage der Repräsentation von „Stadt“ beantworten. Die Grundlage für die Szenenidentifikation bzw. die SzeneTypologie für Berlin und Leipzig bilden qualitative Interviews und Gruppendiskussionen mit je 25 Marktteilnehmern. Diese wurden über minimale und maximal Kontrastierungsregeln für eine fallrekonstruktiven Analyse aufbereitet (Matthiesen 2002, 119 - 125). Im Zentrum der Interpretation stand die Frage, wie Marktteilnehmer branchenspezifische Interaktionskontexte bewerten, wie sie den Zugang zu diesen Materialisierungen informeller Netzwerke beschreiben und welche Strategien sich hinsichtlich der Positionierung in einem neuen Feld zu erkennen geben.

\section{Kontextbeschreibung}

Vor dem Hintergrund der Frage nach der sozialräumlichen Verfasstheit der Kultur- und Kreativwirtschaft gibt sich die Situation in Ostdeutschland als paradox zu erkennen: Deindustrialisierung, Abwanderung und demographischer Wandel, führte seit $1990 \mathrm{zu}$ massivem Wohnungsleerstand., hat aber auch punktuell neue Experimentierräume eröffnet: Galerien, Ateliers oder temporäre Projekte sind Orte, die von Kreativen für sich und ihr unternehmerisches Handeln genutzt und dabei wieder in den ökonomischen Verwertungskreislauf eingebracht werden (Steets 2005, 108-112). An diesen Orten manifestieren sich soziale Netzwerke der Kreativen, die gleichsam eine Machbarkeitsbehauptung abgeben: In Zeiten einer globalen Wirtschaftskrise, schrumpfenden produzierenden Gewerbes, Ideenlosigkeit etablierter und administrativer Funktionsträger sowie zunehmenden Individualisierungsanforderungen, reklamieren gerade junge Kreative neue Formen der Zusammenarbeit sowie des gemeinsamen (wirtschaftlichen) Handelns (Friebe / Ramge, 2008). Dabei steht der ökonomische Profit nicht im Vordergrund, sondern die Umsetzung individueller Gestaltungsabsichten in Kollektiven, um darüber hinaus wiederum Autonomie über Zeit und Sozialität sowie über symbolische Gewinne zu erzielen. 


\section{Unternehmerische Szenen in Leipzig}

Leipzig galt lange Zeit als die heimliche Kulturhauptstadt der DDR, sie war Sammelbecken für diverse Subkulturen und kreative Akteure (Farin 2002, 154). So prägten verschiedene Literaten, bildende Künstler bzw. Punk-Bands das Bild und die Atmosphäre der Stadt (Bismarck 2005). Die politische Wende 1989 bzw. die in den 1990er-Jahren einsetzende Globalisierung führten zu einer Neuformierung der Netzwerke und Szenen der Kulturproduzenten auch in Leipzig. Zur Bewältigung dieser Veränderungen waren gerade informelle Kommunikationsnetze von zentraler Bedeutung. Über 1996 zumeist kleine und mittelständische Unternehmen der Kultur- und Kreativwirtschaft erwirtschafteten im Jahr 2006 einen Umsatz von über 1,5 Mrd. Euro. Die Kultur- und Kreativwirtschaft ist mit ihren rund 10.500 Erwerbstätigen ein relevanter Arbeitsmarktfaktor. Leipzig stellt dabei ein überregionales Zentrum der sächsischen Kultur- und Kreativwirtschaft dar und weist $35 \%$ aller sächsischen Betriebe im Verlagsgewerbe, $23 \%$ in der Werbung, 36\% in der Filmwirtschaft und $34 \%$ aller Journalistenbüros auf (SMWA, 2008, S. 17).

\section{Raumpraktiken der Design- und Kunstszene}

Die mikroräumlichen Praktiken der Kultur- und Kreativszenen Leipzigs sind im Kontext einer „perforierten Stadt“ zu verstehen. Verfall und Leerstand wie v.a. innerstädtische Modernisierung prägt das Stadtbild. Sie entspricht nicht mehr dem klassisch europäischen Bild einer verdichteten Stadtstruktur (Steets 2008: 167). Die mikroräumlichen Praktiken der Leipziger Design- bzw. Kunstszene sollen im Folgenden am Beispiel der Designers' Open bzw. anhand der Baumwollspinnerei beschrieben werden: Die Designers' Open wurde 2004 nach dem Vorbild der Kölner Designmesse von den Designern Jan Hartmann und Andreas Neubert vom Studio Hartensteiner mit dem Ziel initiiert, eine Designplattform für ganz Sachsen zu schaffen. Sie ist heute ein zentraler Branchentreff, eine Entdeckermesse für junge nationale und internationale Designer sowie ein Kommunikationsforum. Die Akteure der Designszene zeigen eine Raumpolitik, die mit auf die instabilen Raumsituation der Stadt reagiert: So waren 2007 die Hauptausstellungsräume der Designers' Open ehemalige und momentan leerstehende Waren- und Ausstellungshäuser in innerstädtischen, zentralen Räumen. Im Jahr 2008 dynamisierten sie ihre dezentrale Praxis der Raumnutzung und suchten sich neue kleinräumige Veranstaltungsstätten, die über den Kernstadtbereich verteilt waren. So wurde die gesamte Innenstadt zur Ausstellungsfläche. Diese enge aufeinander bezogene Konzeption der Räume steht für das äußerst wichtige auf Netzwerken basierende Zu- 
sammenarbeiten der Szene. Zudem verkörpert die Aneignung zentraler Orte in Leipzig den Wunsch nach stärkerer Präsenz im Bild der Stadt. Auch die Marketingmaßnahmen für die Designers' Open folgen der Prämisse, möglichst viele Interessierte anzuziehen; neben den Ausstellern auch das breite Publikum, um so eine höhere Nachfrage und Interesse für die Leipziger Designszene zu erreichen. Es werden Anzeigen in den städtischen Medien geschaltet, Flyer und Mails verschickt, die sich nicht ausschließlich auf Szene-Angehörige beziehen.

Anders als die temporäre und dezentrale Praxis der Designzene, stellt sich die Leipziger Kunstszene dar, die mit einer anderen Standort- und Raumnutzungspolitik auf die städtischen Raumbedingungen frühzeitig reagierte. Mit der Baumwollspinnerei im Leipziger Westen wurde ein Raumnutzungsmodell geschaffen, das im Laufe der Jahre modellhaften Charakter erhielt und von anderen Kreativen in Leipzig immer wieder praktiziert wurde: die kostengünstige Aneignung ehemaliger Industrieflächen als Schaffensräume und gleichzeitig die Herstellung und Verstetigung von sozialen Netzwerken. Der Boom der stilbildenden „Neuen Leipziger Schule“ stellt dabei einen wichtigen Ausgangspunkt für die Entwicklung des Areals der ehemaligen Baumwollspinnerei dar. Auf dem Gelände, das mittlerweile professionell geführt und geleitet wird, arbeiten gegenwärtig rund 80 Künstler; 2005 zogen die wichtigsten Kunstgalerien Leipzigs auf dem Gelände ein, zudem haben sich eine Reihe unterschiedlicher Gewerbebetriebe angesiedelt (Steets 2008, 174-177).

Ihre Arbeits- und Ausstellungsräume haben die Kreativen in der Baumwollspinnerei bislang nur notdürftig in Stand gesetzt, um ein halbwegs gutes Arbeiten zu ermöglichen; gleichzeitig soll die Haptik des Ortes, seine Symbolkraft nichts verlieren. Szenegänger und Besucher sollen spüren, dass es ein offener und unfertiger Ort ist, der frei und kreativ bespielt werden soll und kann. Das Unfertige der Baumwollspinnerei weist aber auch auf temporäre Aufenthalte und Raumaneignungspraktiken hin. Die Akteure machen nun Station in Leipzig, sind dabei auch fest im Stadtteilmilieu verankert, halten sich aber neue Wege, die weg aus Leipzig führen, offen.

Im Gegensatz zur Leipziger Designszene funktionieren die mikroräumlichen Praktiken der Leipziger Kunstszene ähnlich wie in Berlin: bei den jährlichen Galerierundgängen wird der Ort inszeniert und symbolisch verdichtet und spricht dadurch Szenegänger und -kenner an. Auf subtile Art und Weise wird sozialer Ausschluss gelenkt, sei es durch die Ankündigung von Partys und Ausstellungen nur via E-Mail-Verteiler oder der Verkauf von Kunstwerken nur an bestimmte, sich als SzeneAngehörige zu erkennen gebende Personen. 


\section{Unternehmerische Szenen in Berlin}

Über 22.900 zumeist kleine und mittelständische Unternehmen der Kultur- und Kreativwirtschaft erwirtschafteten 2006 einen Umsatz von über 17,5 Mrd. Euro und erreichten damit einen Umsatzanteil von über $21 \%$ am Bruttoinlandsprodukt der Berliner Wirtschaft. Die Kultur- und Kreativwirtschaft stellt mit ihren rund 160.500 Erwerbstätigen einen relevanten Arbeitsmarktfaktor am Standort dar. Über 10\% der Berliner Erwerbstätigen arbeiten in den verschiedenen Branchen der Kulturund Kreativwirtschaft (Senatsverwaltung für Wirtschaft, Technologie und Frauen 2008, S. 24). Jährliche Messen und regelmäßig bespielte Ausstellungsorte unterstreichen dies, sie sind auch ein erster Schlüssel, um Prozesse der Imagebildung Berlins zu begründen.

Berlin hat in der Situation einer stetig anwachsenden sozioökonomischen Strukturkrise seit dem Jahr 2000 versucht mit dem Schlagwort „neues Unternehmertum“ ökonomische Dynamiken zu entwickeln. Kreativen Berufen wurde dabei von Seiten der Politik und der Wirtschaft eine zukunftsweisende Rolle für die auf Information und Innovation basierenden Ökonomien zugewiesen. 1998 skizzierte daher der Soziologe Heinz Bude erste Merkmale und Ausprägungen dieser sich neu ausgestaltenden „Generation Berlin“ (Bude 2001). Dabei versuchte er den diskursiven Boden zu bestellen, der maßgeblich bestimmt war durch neue Praktiken und Artikulationen von Akteuren, die nun als „,neue kulturelle Unternehmer“ betitelt werden. Eine der kulturellen und städtischen Schlüsselentwicklungen der Berliner Nachwendezeit wird durch die Präsenz von neuen hybriden, nämlich kulturellen sowie unternehmerischen Szenen bestimmt. Neue kulturelle sowie unternehmerische Szenen sind dabei Sinnstifter einer dynamischen Alltagskultur, in der auch das Wissen um sowie die Produktion und die Organisation von sozialen und physischen Räumen sowie die symbolischleibliche Zugehörigkeit von zentraler Rolle sind. Denn in und mit diesen Räumen können Identitäten geformt und gegeneinander abgegrenzt werden.

Relevanz hinsichtlich unternehmerischer Praxis erfährt dieses Thema in Bezug auf Berlin nicht nur bei der Wirtschaftsförderung und der Beschäftigungspolitik, sondern zusätzlich auch auf der Imageebene der Stadt: Leitbegriffe wie „Capital of Talents“ oder „Young Creative Industries" greifen - ähnlich wie in anderen Metropolregionen - eines der dynamischsten Potenziale urbaner Ökonomie auf. Hinter Begriffen wie Çool Britannia“ und „Generation Berlin“ stehen bisher unhinterfragte Zusammenhangsformen von neuen, zunächst weitestgehend als räumlich flexibel verstandenen Professionsgruppen und Symbolträgern sowie deren temporäre Verankerungen in neuen, räumlich lokalen Struktur- 
mustern mit neuen Raumbedürfnissen und Raumaneignungs- wie Differenzierungsstrategien.

\section{Raumpraktiken der Design- und Kunstszene}

Die mikroräumlichen Praktiken der Design- und Kunstszene sind im Kontext einer Stadt zu lesen, deren Terrain kulturell und symbolisch umkämpft ist. Dieser kulturelle Widerstreit wird über die Deutungshoheit und die Aneignungspraktiken von Orten geführt. Insbesondere ausbleibende Wachstumseffekte und Investitionen sowie überzogene Planungserwartungen haben junge Akteursgruppen aus dem Bereich expressiver Berufsgruppen motiviert, sich auf ausgewiesenen Terrains der Global Player visuell in Szene zu setzen. Mit temporären Aktionen (z. B. Strandbars) werden Nutzungsansprüche des Städtischen reklamiert. Die neuen kulturellen Unternehmer spielen dabei eine Schlüsselrolle: Sie legen aufgrund ihrer Wissens- und Erfahrungsressourcen über den Zusammenhang von Vergemeinschaftungen - Stichwort Events - Ortspraktiken an den Tag, die feine Distinktionsangebote nicht nur bereitstellen. Vielmehr gehen sie - gezwungenermaßen - von einem eigenen Territorialverständnis aus und entwickeln und praktizieren so letztlich eine andere Theorie des Ortes. Diese Territorialpraxis ist nicht nur ein gekonntes Jonglieren mit Symbolen, Ästhetiken und semiotischen Materialien, vielmehr manifestiert sich in ihr gesellschaftliche Teilhabe oder sozialer Ausschluss, sei es in Milieus, Szenen oder anderen Vergemeinschaftungen. Diese Materialien sind Ausdrucksgestaltungen, Oberflächenphänomene, hinter denen der Anspruch steht, sich zwanglos zu vergemeinschaften und sich dieser Vergemeinschaftung immer wieder zu vergewissern.

Im Kern zeigen die mikroräumlichen Strategien der Protagonisten die Fähigkeit auf, immobilen und vordergründig klar programmierten Raum temporären Veränderung und Umprogrammierungen aussetzen. Büroräume werden zu organisatorischen Werkstätten und Clubs, diese temporär verlagert und strategisch mit kommunikativen Attraktionspotenzialen ausgerichtet. Der notwendige organisatorische Werkstattcharakter dieser Unternehmungen zeigt kommunikative Strategien, den Mikrostandort „Büro“ als Knoten für fluide soziale Vergemeinschaftungen und künstlerische Szene zu bespielen.

Der hier untersuchte Ort - eine Büronutzung des Unternehmens "Greige“ - zeigt, wie diese Akteure eine eigenwillige und zugleich spielerische Politik der Aufmerksamkeit an den Tag legen. Um ihn in den Köpfen von Szenegängern existent werden zu lassen, zeigen sie eine spezifische Ortspolitik, die soziale Zugehörigkeit neu verhandelt. Ihr Ort ist z. B. der Treffpunkt einer offenen, aber klar umrissenen Gruppe von Freunden, Kollegen, Konkurrenten, Interessierten und Neugierigen. Der 
Zugang zu diesem Ort und seine Wahrnehmung werden durch eine Politik gelenkt, die Ähnlichkeiten mit der eines Clubs aufweist. Doch der bekannte Selektionsmechanismus eines Clubs - Zurückweisung an der Tür durch Türsteher - nimmt in diesem Fall weitaus subtilere Formen an. Verschiedene Medien, wie Mund-zu-Mund-Propaganda, Mailinglisten und Flyer, sichern die gezielte Verbreitung von Informationen über anstehende Events, Ausstellungseröffnungen oder auch neue Produkte. Neben dieser Informationspolitik arbeitet man aber auch immer wieder daran, den Ort in der Versenkung verschwinden zu lassen, um die soziale Gefolgschaft klein zu halten.

In diesem Fall lässt sich ein Spiel erkennen, das die Besucher ausbalanciert, den Ort tarnt und ihn anschließend wieder ins Bewusstsein rufen will. Die Akteure arbeiten ohne ein Jahresprogramm und kündigen ihre Kunstausstellungen kurzfristig an, indem sie die Einladung per Mailingliste an ausgewählte Interessierte und Freunde aus der Berliner, Kölner, nationalen und der europäischen Kunstszene verschicken. Die in der Kopfzeile der E-Mail abzulesende Zugehörigkeit zu der damit offensichtlich gewordenen „Szene“ ist das Aus- und Einschlusskriterium, um das sich offen ausgesprochen aber niemand kümmert.

Die zunächst erstaunlich und widersprüchlich erscheinende Strategie des Verbergens greift eine Haltung auf, die an die alte sozialistische Dienstleistungsmentalität erinnert: Der Kunde ist nicht König und das Geschäft vordergründig egal. Diese Strategie kommt auch äußerlich zum Einsatz: Der Ort gibt optisch nach außen nicht zu erkennen, welche Events sich in seinem Inneren abspielen. Nur Ortskundige nehmen ihn als Ort von Veranstaltungen wahr und können ihn „lesen“. Indem er sich mittels einer solchen Versteckpolitik im städtischen Raum positioniert, wird nicht nur soziale Differenz erzeugt, sondern vor allem die breite Masse fern gehalten.

Wenn eifrige Suchende den Ort dennoch finden, tritt ein weiteres, subtil erzeugtes Differenzierungskriterium in Kraft. Auf den beispielsweise nach Eröffnungen stattfindenden Partys werden den anwesenden Gästen durch die ausgestellte Kunst und die elektronische Musik verschiedene Identifikationsmuster angeboten. Die Zuordnung zu diesen, basierend auf der Erfahr- und Lesbarkeit der Performance ermöglicht erst das einprägsame Erlebnis der Teilnahme an einem Event. Darin liegt die subtile Ausgrenzungsstrategie: Niemand wird des Ortes verwiesen, jeder vielmehr zugelassen, doch nur wenige werden integriert. Und auch diese erste Integration ist eine Aufforderung, sich der Zugehörigkeit permanent $\mathrm{zu}$ versichern.

Das Verständnis dieser mikroterritorialen Praxis ist nur vordergründig erstaunlich, sollte man doch davon ausgehen, dass die ohnehin ma- 
gere Auftragslage und der immense Konkurrenzkampf dazu führen, dass alle Kräfte darauf gebündelt werden, direkte Produkte zu entwickeln und zu verkaufen. In dieser Ortspraxis zeigt sich aber, dass die bewusste temporäre und flexible Gestaltung von Orten notwendig ist, um sich über diesen Nukleus „Ort“ als Gestalter und Attraktor von sozialen Formationen zu zeigen. In diesen Mikroarenen muss sich demzufolge erst ein Produkt, ein Stil, ein Trend kurz: eine kulturelle Innovation - behaupten, sie muss die Testphase auf Partys, Ausstellungen und Eröffnungen bestehen, um erstens Verbreitung zu erlangen und zweitens temporäres Distinktionsmaterial für Vergemeinschaftungsprozesse bereitzustellen. Rückwirkend brauchen Szenen und Milieus diese instabilen und kurzfristigen Manifestationsarenen, d. h. Orte, an denen die latenten Beziehungen expliziert werden, an denen sich eine Szene zeigt, erfährt, informiert und durch die Erfahrung, den Austausch transformieren und modifizieren kann.

\section{Variationen und Typiken von Szenen in Leipzig und Berlin}

In Leipzig und Berlin lassen sich drei relevante unternehmerische Variationen und Typiken von Szenen identifizieren:

1. Unternehmerische Gewinnerszenen: In Leipzig zeigen sich insbesondere (stadt-)bekannte Multiplikatoren, z. B. Agenturbesitzer im Werbe-, Design- und Galeriebereich, die hohe Ausstrahlungseffekte auf andere Szenen haben und relativ stabile Kleinunternehmen führen. Sie weisen eine hohe Transferkompetenz zwischen Teilmärkten auf. Oftmals variieren sie Profit- und Gewinnstreben mit Investitionen in Non-Profit-Aktivitäten (öffentliche Kunst), wodurch sie eine wichtige Funktion für Kulturstandorte aufweisen (Bsp. Designszene). In Berlin dagegen sind die Multiplikatoren, z. B. Modelabelbesitzer und Galeristen, transnational ausgerichtet und haben dadurch hohe Ausstrahlungseffekte auf den gesamten Markt. Sie sind gleichwohl auf lokale Netzwerke von Kleinstunternehmern angewiesen. Diese unternehmerischen Eliten weisen eine hohe Standortungebundenheit auf. Starkes Profit- und Gewinnstreben mit Investitionen in anderen Profitbereichen (Kunst), wodurch sie eine wichtige Funktion für den gesamten Kulturstandort Berlin aufweisen.

2. In beiden Städten zeigen sich aber auch junge mikrounternehmerische Szenen: Sie sind sehr flüchtige und in schnell umbrechenden Netzwerken von jungen Unternehmern im Übergangsbereich zwischen Qualifikationsbeendigung und existenzsichernden Erwerbsoptionen zu finden. Sie weisen starke Verankerung in sozialen Stadtteilmilieus (Nischenstruktur), um oftmals prekäre Existenzen zu meistern. 
3. Berlin weist, anders als Leipzig, etablierte „mittelständsähnliche“ unternehmerische Szenen auf, die einen höheren Formalisierungsgrad als die Nischenszenen aufweisen. Aufgrund der Größe der Metropole Berlin zeigt sich bei ihnen ein hohes Maß an lokal-gebundenen Wissens-, Produktions- und Verfahrenskompetenzen.

\section{Szene - ein wirtschafts- und kulturgeographisches Konzept?}

Die vorangegangenen Ausführungen diagnostizierten den bisherigen Gebrauch sowie die gestiegene Benutzung des Begriffs Szene in ausgewählten sozialwissenschaftlichen Disziplinen sowie im anwendungsorientierten Bereich (z. B. Politikberatung). Die hier vorgenommene Darstellung konzentrierte sich dabei auf die Anwendung des Begriffs Szene auf Ort, Raum und Stadt sowie genauer auf die Verwendung in der Kultur- und Kreativwirtschaft. Szenen werden in diesem jungen Segment als Inkubator für neue kulturelle Wertschöpfungsprozesse angesprochen, die sodann die regionale Wettbewerbsfähigkeit positiv beeinflussen können. Die folgende Zusammenfassung fokussiert auf die Verbindung von Szenen zu Raum und Ort sowie auf ortsspezifische Artikulationen von Szenen.

\section{Vergemeinschaftungsarbeit}

Die untersuchten Szenen vermitteln und aktualisieren expressive Werte wie Selbstverwirklichung, Individualität, Autonomie und Kreativität (Koppetsch/Burkart 2002). Die dabei artikulierten Werte und Wertbindungen müssen ständig reproduziert, situativ mobilisiert und zeitlich eng rhythmisiert in sozialen Arenen überprüft werden. Szenen geben sodann eine Individualisierungsaufforderung $\mathrm{zu}$ erkennen, die eingebunden ist in eine erlebnisorientierte Kulturpraxis, die im Grunde genommen alles andere als ein spaßbetontes Erlebnis ist. Vielmehr ist Vergemeinschaftungsarbeit angesagt.

Unternehmerische Szenen artikulieren sich in neuen projektbasierten Kooperationsformen mit veränderten räumlichen Praxen und Ortspolitiken. In diesen neuen Praktiken zeigt sich ein spielerischer Umgang mit "Ort" und „Raum“. In ihnen bündeln sich, vermittelt durch spezifische Verortungsstrategien, zeitweise immer wieder verschiedene Szenen. Orte sind damit das Terrain in der postindustriellen Stadt, um die heterogene Gruppen, Gemeinschaften und Gesinnungen sowie auch unternehmerische Szenen symbolisch kämpfen, um ihren Mitgliedern durch die physische Präsenz im städtischen Raum eine Einbettungs- 
und Verortungsmöglichkeit zu gewährleisten. Die dabei verwendeten kulturellen Codierungen, urbanen Mythen und lokalen Narrative werden oftmals von Szenen an ausgesuchten Orten im Urbanen mitgestaltet und formuliert.

Szenen sind demzufolge neue wie auch zukünftige soziale Schaltzellen in einer individualisierten Gesellschaft, an denen Neuformierungen temporär getestet werden können. Hierbei zeigen sich räumliche Praktiken und Bewegungsmuster, die sich ganz bewusst gegen die Strategien einer Geografie der Zentralität abheben und abgrenzen: Diese am Beispiel von Leipzig und Berlin vorgestellten Geografien des Ephemeren, des Temporären und Selektiven konkurrieren mit einer Geografie der symbolisch-touristischen sowie konsumorientierten Zentralität. Die dabei verwendeten Praktiken sind im de Certeau'schen Sinn als Taktiken anzusprechen, weil sich an ihnen subtile Praktiken des temporären Widerstandes gegen machtvolle Markt- und Politikstrukturen samt ihrer disziplinierenden Ordnungen zeigen lassen. Diese Politikpraxis ist aber keine klassisch gegenpolitische, vielmehr eine bewusst subtile, um einen (Wahrnehmungs-)Ort der Widersprüchlichkeit zu schaffen. Die dabei zutage tretenden räumlichen Ambivalenzen und Brüche bieten den Akteuren ausreichend Material, um sich daran zu reiben, sich ihrer selbst zu vergewissern oder zu spiegeln; Die sich an diesen Orten manifestierende Szene formiert sich demzufolge erst an und mit den Ausstrahlungskräften dieses Ortes, an dem sich eine emotionale Steigerung für Individuen zeigen.

\section{Szenen und Zwischenorte}

Die vorangegangenen Ausführungen beleuchteten das Wechselverhältnis zwischen konzeptionellen Ansätzen neuer Vergemeinschaftungen und Raum. Einen wesentlichen Beitrag zum Verständnis dieses Wechselverhältnisses hat die Raumsoziologin Silke Steets am Fall von Leipzig vorgelegt. Ihrer Perspektive zufolge dient (städtischer) Raum kulturellen Trägergruppen als Mittel, um durch räumliche Mikropolitiken Zwischenorte zu erzeugen (Steets 2005). Steets betrachtet diese städtischen Räume aus der Perspektive von Künstlern, die in Zeiten schrumpfender Städte „freigesetzte“ Orte, deren ursprünglicher Zweck verloren ging (Industrieanlagen), neu nutzen und in Form von Clubs, Bars bzw. Galerien aufwerten. Durch diese Form der Raumaneignung entstehen vergängliche Räume aus der Interaktion zwischen materiell-physischen (An)Ordnungen, narrativen, bildlichen Aufladungen der (An)Ordnungen und Handlungen der Nutzer. Diese Räume befinden sich in einem Zwischen-Zustand, zwischen Privatheit und Öffentlichkeit, Legalität und Illegalität, Markt und Kritik (Steets 2005: 115-118). 
Der Prozess der Entstehung vergänglicher Räume beinhaltet die Verunsicherung des Publikums durch eine diskursive und tatsächliche Vermischung unterschiedlicher Veranstaltungsformen, wie Workshop und Café; ein hohes Maß an Selbstorganisation der Künstler; wenig Handlungsstrukturierung durch lückenhaftes Spacing; die Komplettierung des Raums durch die Mitarbeit verschiedener Akteure; die Inklusion bzw. Exklusion des Raumes durch implizite Lesbarkeit der (An)Ordnungen (Löw 2001, 98-99). Letzteres schränkt die anvisierte Offenheit dieser Räume jedoch ein, da sie ein gewisses szenetypisches Know-how voraussetzen.

\section{Eigenlogiken}

Unternehmerische Szenen, so zeigten die beiden Fallrekonstruktionen, basieren im Wesentlichen auf sozial-räumlichen Differenzierungspraktiken. Dabei folgen sie aber nicht universellen Regeln, sondern geben Eigenlogik des Städtischen zu erkennen (Löw 2008). Es existieren Regeln und Gewissheiten über das Besondere einer Stadt, über das Funktionieren von bestimmten Ritualen, Sprachcodes und Weltanschauungen. Eigenlogik kann als die Grammatik einer Stadt und eines Ortes beschrieben werden, durch die festgelegt ist, was in dieser Stadt überhaupt möglich ist, worin also im Alltäglichen das Spezifische liegt (Löw 2008: 43).

Dies gilt im Besonderen für die Orte aber auch Arbeitskontexte der Kultur- und Kreativwirtschaft. Orte geben sich erst durch soziale und kommunikative Prozesse zu erkennen, es bedarf also einer sozialen Verdichtung, gleichsam körperlicher Präsenz von Szeneteilnehmern, da eine sinnliche Erfahrbarkeit zwischen Akteuren notwendig ist, um z. B. Leipzig als bedeutsamen Ort überhaupt erst kollektiv wahrnehmbar zu machen. Diese Orte haben aber für Szenegänger den Zweck, Zugänge zu Markt auf der Grundlage kultureller Netzwerke zu formieren. KreativAkteure organisieren Ausstellungen, Produktpräsentationen, Vernissagen, Finissagen, Feiern etc. also kurzzeitige Ereignisse, deren Performativität Sozialräume mitstrukturiert. Wichtig ist dabei, dass es gilt die Sprach- und Verhaltenscodes zu beherrschen, mit denen Zugang zu diesen sozialen Orten und Ereignissen erst möglich werden. Das „,,look ,feel' of the location" (Helbrecht 2004, 200) entscheidet über den Erfolg. Orte haben also eine Eigenlogik, die sich in eben örtlich spezifischen und nicht universell einheitlichen Formen der Vergemeinschaftungen zu erkennen geben.

Sie inszenieren Ereignisse, die Orte als soziales Feld auf einer städtischen Karte auftauchen lassen. Diese Aktivitäten zielen darauf ab, soziale Gefolgschaften sowie Handlungssicherheit zu erlangen. Sie erzeu- 
gen soziale Intensitäten und erregungsreiche Verdichtungen, die Aufmerksamkeiten nach sich ziehen und als Effekt darauf abzielen, Trends, Stile und Codes, kurz: symbolische Produkte, in soziale Beziehungsnetze hineinzufiltern. Diese Ortspolitiken und das kann man insbesondere im Bereich Design, Musik, Literatur und Mode beobachten, zielen darauf ab, die Produkte der Kultur- und Kreativwirtschaft - also Sounds, Bilder, Texte, Techniken, Grafiken - auf ihre performative Wirkung hin zu testen. Der soziale und performative Prozess des Testens vollzieht sich in den temporären und transitorischen Räumen dieser Kreativszenen. Berlin hatte diese Raumangebote, so dass sich spezifische Kompetenzen der Ortsinszenierung entfalten konnten. Leipzig entdeckt diese Raumoptionen im größeren Stil erst in den letzten Jahren. An diesen Orten können sich die performativen Qualitäten von immateriellen und symbolischen Produkten in Netzwerksozialitäten entfalten und sodann verhandelt werden. Denn erst dann kann das kommunikative Vokabular generiert werden, mit dem dann ausgesprochen wird, worin die Qualität eines Gegenstandes, also eines zum Testen bereitgestellten Produktes, liegt. Die Fallrekonstruktionen zeigen, dass es zwei zentrale wenngleich diametrale Prozesse gegenüberstehen: 1. Die Wichtigkeit des sozialen eigenlogischen Ortes als Zugang zu Marktprozessen. 2. Die Flüchtigkeit dieses sozialen Kreativfeldes.

\section{Zusammenfassende Darstellung des Konzepts Szene}

Wenn Szenen nicht nur als zeitdiagnostische Figur der Posttraditionalisierung der Gesellschaft Anwendung finden, sondern auf unternehmerische Kontexte der Symbolproduktion übertragbar werden, dann gilt es zunächst anzuerkennen, dass der Begriff Szene aus dem Kernbereich seiner Verwendung (Jugend- und Subkulturen) herausgelöst und auf unerwartete gesellschaftliche Handlungsfelder (Wirtschaft) angewandt werden kann. Diese Anwendung ist dann nicht eine, die soziales neben räumlichen Determinanten stellt, sondern eine, die dem so genannten spatial turn in den Sozialwissenschaften Rechnung trägt: soziale Praktiken finden nicht in einer Stadt statt, sondern sind Ausdruck des spezifisch Städtischen.

Während Ronald Hitzler das Phänomen Szenen weitestgehend raumlos konzipiert und letztlich Soziales neben Raum stellt, zeigen sich folgenreiche Konsequenzen, wenn Szenen in ihrem Konstruktions- und Aufführungscharakter in Wechselwirkung zu Raum gesetzt werden. Damit kann eine erste Kontextdeterminante angesprochen werden. Eine zweite ist in der Hitzler'schen sowie der wissenssoziologischen Perspektive angelegt: Das Konzept und Verständnis von Szene ist anwendbar auf den Kontext unternehmerisches Handeln und somit auf Formen der 
Interaktion in bestimmten Märkten, d. h. auf unternehmerische Szene in der Kultur- und Kreativwirtschaft. Doch auch hier gilt es die durch den spatial turn hervorgehobene Kontextdimension als eine erklärende und differenzierende anzusprechen, um von da aus nach den kontextspezifischen Artikulationen von unternehmerischen Szenen in Teilmärkten der Kultur- und Kreativwirtschaft zu fragen.

1. Szene ist als sozialräumliche Strukturkategorie anzusprechen. Sie weist flexible, ereignisbasierte Interaktionsformen mit erhöhter Binnenkommunikation auf, die durch implizites, geteiltes Wissen um gemeinsame temporäre Praxisformen geprägt ist.

2. Unternehmerische Szenen in kultur- und kreativwirtschaftlichen Bereichen eröffnen nicht nur Erwerbsoptionen, sie strukturieren aufgrund ihrer Raumpraktiken unternehmerische Beziehungsnetze.

3. Szenen im soziologischen Sinn ausschließlich als Inszenierungspraktiken anzusprechen, wie dies bspw. Ronald Hitzler macht, heißt, Soziales entweder neben Raum stellen und Raum als Behälterraum neben Soziales zu denken oder ihn gänzlich ignorieren. Ebenso erscheint es wenig zielführend mittels eines Begriffs des theatralisch Szenischen die Unmittelbarkeit des Städtischen anzusprechen. Dabei wird die strukturierende Wirkung von Szenewissen und Szeneerfahrungen auf sozialräumlicher Ebene außer Acht gelassen.

4. Szene strukturiert sich um Organisations- und Professionseliten, es sind keine homogenen Gesellungsgebilde.

5. Das Potential dieses Szeneverständnisses als analytisches Konzept des Räumlichen besteht gerade darin, dass die durch Anordnungspraktiken initiierten informellen Vergemeinschaftungen auf ihre raumstrukturierenden Effekte hin überprüft werden können.

\section{Literaturverzeichnis}

Berking, H./Löw, M. (2005): Wenn New York nicht Wanne-Eickel ist... Über Städte als Wissensobjekt der Soziologie. In: H. Berking/M. Löw (Hg.), Die Wirklichkeit der Städte. Baden-Baden, Nomos, S. 9-24.

Bismarck, B. / Koch, A. (2005): Beyond education. Kunst, Ausbildung, Arbeit und Ökonomie, Frankfurt am Main, Revolver.

Blum, A. (2001): Scenes. In: J. Marchessault/W. Straw (Hg.), Public (Special Issue of Public). New York / London, S. 7-36.

- (2003): The imaginative structure of the city, Montreal, McGill-Queen's University Press. 
BMWi 2009: Gesamtwirtschaftliche Perspektiven der Kultur- und Kreativwirtschaft in Deutschland. Berlin: Bundesministerium für Wirtschaft.

Bogner, A./Menz, W. (2002a): Das theoriegenerierende Experteninterview. Erkenntnisinteresse, Wissensformen, Interaktion. In: A. Bogner / B. Littig / W. Menz (Hg.), Das Experteninterview - Theorie, Methode, Anwendung. Wiesbaden, Verlag für Sozialwissenschaften, S. 33-70.

- (2002b): Expertenwissen und Forschungspraxis: die modernisierungstheoretische und die methodische Debatte um die Experten. In: A. Bogner/B. Littig/W. Menz (Hg.), Das Experteninterview - Theorie, Methode, Anwendung. Wiesbaden, Verlag für Sozialwissenschaften, S. 7-29.

Bude, H. (2001): Generation Berlin, Berlin, Merve-Verlag.

Certeau, M. D. (1988): Kunst des Handelns, Berlin, Merve-Verlag.

Cohen, S. (1997): Symbols of Trouble [1980]. In: K. Gelder/S. Thornton (Hg.), The subcultures reader. New York, Routledge, S. 146-162.

Dürrschmidt, J. (2000): Everyday lives in the global city. The delinking of locale and milieu, London $\mathrm{u}$. a., Routledge.

Ertel, R. (2006): Daten und Fakten zur Kulturwirtschaft. In: Aus Politik und Zeitgeschichte, $34-35$, S. $17-23$.

Farin, K. (2002): Generation-kick.de. Jugendsubkulturen heute, München, Beck

Friebe, H. / Ramge, T. (2008): Marke Eigenbau: der Aufstand der Massen gegen die Massenproduktion. Frankfurt am Main [u. a.]: Campus-Verlag.

Froschauer, U./Lueger, M. (2002): ExpertInnengespräche in der interpretativen Organisationsforschung. In: A. Bogner / B. Littig/W. Menz (Hg.), Das Experteninterview - Theorie, Methode, Anwendung. Wiesbaden, Verlag für Sozialwissenschaften, S. 223-240.

Gelder, K. (1997): Introduction to part two [The Birmingham tradition and cultural studies]. In: K. Gelder, K./S. Thornton (Hg.), The subcultures Reader. New York, Routledge, S. $83-89$.

Gelder, K. / Thornton, S. (1997): The subcultures reader, London / New York, Routledge.

Grabher, G. (2006): Handelsrouten, Umwege, gefährliche Kreuzungen: die Wege des Netzwerkbegriffs von der Wirtschaftssoziologie in die Wirtschaftsgeographie (und retour). In: C. Berndt/J. Glückler (Hg.), Denkanstösse zu einer anderen Geographie der Ökonomie. Bielefeld, Transcript-Verlag, S. 77 - 110.

Hasse, J. (2002): Zum Verhältnis von Stadt und Atmosphäre. Wo sind die Räume der Urbanität? In: J. Hasse (Hg.), Subjektivität in der Stadtforschung Frankfurt am Main, Selbstverlag des Instituts für Didaktik der Geographie der Johann Wolfgang Goethe-Universität Frankfurt am Main (Natur-Raum-Gesellschaft 3), S. $19-40$.

- (2008): Die „Stadt“ als Situation. In: M. Großheim (Hg.), Neue Phänomenologie zwischen Praxis und Theorie. Freiburg und München, S. 224-237. 
Helbrecht, I. (2004): Bare Geographies in Knowledge Societies - Creative Cities as Text and Piece of Art: Two Eyes, One Vision. In: Built environment, 30, 3, S. $194-203$.

Hesse, M. / Lange, B. (2007): Kreative Industrien - Magma und Mantra der Stadtentwicklung. Das Beispiel Berlin. In: Kommune, 2, S. 64-69.

Hitzler, R. / Bucher, T. / Niederbacher, A. (2001): Leben in Szenen. Formen jugendlicher Vergemeinschaftung heute, Opladen, Leske + Budrich.

Hospers, G. J. (2003): Creative cities: Breeding places in the knowledge economy. In: Knowledge, Technology and Policy, 16, 3, S. 143-162.

Janson, A. (2004): Kapazität des Szenischen. In: Der Architekt, 5 / 6, S. 20 -22.

Klein, G. (Hrsg.) (2005a): Stadt. Szenen. Künstlerische Produktionen und theoretische Positionen. Wien, Passagen-Verlag.

- (2005b): Die Stadt als Szene. Zur Einführung. In: G. Klein (Hg.), Stadt. Szenen. Künstlerische Produktionen und theoretische Positionen. Wien, Passagen-Verlag, S. $13-35$.

Koppetsch, C./Burkart, G. (2002): Werbung und Unternehmensberatung als „Treuhänder“ expressiver Werte? Talcott Parsons' Professionssoziologie und die neuen ökonomischen Kulturvermittler. In: Berliner Journal für Soziologie, 12, 4, S. $531-550$.

Lange, B. (2007): Die Räume der Kreativszenen. Culturepreneurs und ihre Orte in Berlin, Bielefeld, Transcript Verlag.

- (2008): Situativer Urbanismus. In: IBA HAMBURG (Hg.), Kreative Ökonomien. Zur Übersetzbarkeit des Ansatzes auf Stadtteilebene. Dokumentation der Fachtagung am 16. und 17. Juni 2008. Hamburg, S. 159-162.

Lange, B. / Mieg, H. A. (2008): Professionalisierungswege und Konstituierungen von „Märkten“ in den Creative Industries. In: Geographische Zeitschrift, 94, 4, S. $225-242$.

Legnaro, A. (2004): Performanz. In: U. Bröckling, U. (Hg.), Glossar der Gegenwart. Frankfurt am Main, S. 204-209.

Löw, M. (2001): Raumsoziologie, Frankfurt am Main, Suhrkamp.

- (2008): Eigenlogische Strukturen - Differenzen zwischen Städten als konzeptuelle Herausforderung. In: H. Berking / M. Löw (Hg.), Die Eigenlogik der Städte. Neue Wege für die Stadtforschung. Frankfurt am Main, Campus, S. 33-54.

Matthiesen, U. (2002): Zur Methodik sozialräumlicher Milieuanalysen. In: C. Deilmann (Hg.), Zukunft - Wohngebiet. Berlin, S. 119-136.

McRobbie, A. (2005): Wie man in Londons kreativen Kleinunternehmen über die Runden kommt. In: B. Binder/S. Göttsch/W. Kaschuba/K. Vanja (Hg.), Ort Arbeit - Körper. Ethnografie Europäischer Modernen. Münster, Waxmann, S. $81-96$. 
Pfandenhauer, M. (2005): Ethnography of Scenes. Towards a Sociological Lifeworld Analysis of (Post-traditional) Community-building (31 paragraphs). Forum Qualitative Sozialforschung/Forum: Qualitative Social Research (Online Journal). Forum Qualitative Sozialforschung.

Reckwitz, A. (2009). Die Selbstkulturalisierung der Stadt. Zur Transformation moderner Urbanität in der „,creative city“. Mittelweg 36. Zeitschrift des Hamburger Instituts für Sozialforschung 18. Jg. (April / Mai): 2-34.

Rossiter, N. (2007): Organized Networks: Media Theory, Creative Labour, New Institutions, Rotterdam / Amsterdam, NAI Publishers.

Schallberger, P. (2003): Junge Gründerinnen und Gründer: Motive, ökonomisches Denken und Möglichkeiten der Förderung, Bern.

Senatsverwaltung für Wirtschaft, Technologie und Frauen (2008): Kulturwirtschaft in Berlin - Entwicklungen und Potenziale (2. Kulturwirtschaftsbericht). Berlin: Senatsverwaltung für Wirtschaft, Technologie und Frauen.

SMWA (ed.) (2008): 1. Kulturwirtschaftsbericht für den Freistaat Sachsen. Dresden, SMWA (Sächsisches Ministerium für Wirtschaft und Arbeit).

Steets, S. (2005): Doing Leipzig. Räumliche Mikropolitiken des Dazwischen. In: H. Berking / M. Löw (Hg.) Die Wirklichkeit der Städte. Baden Baden, Nomos Verlagsgesellschaft, S. 107-122.

- (2008): „Wir sind die Stadt!“ Kulturelle Netzwerke und die Konstitution städtischer Räume in Leipzig, Frankfurt am Main, Campus.

Weckerle, C. / Gerig, M. / Söndermann, M. (2007): Kreativwirtschaft Schweiz - Daten, Modelle, Szene, Basel, Birkhäuser Verlag.

White, H. C. (2002): Markets from networks: socioeconomic models of production, Princeton u. a., Princeton University Press.

\section{Zusammenfassung}

Der Beitrag präzisiert eine vorherrschende begriffliche Unschärfe in der Sozial-, Kultur- und Wirtschaftsgeographie. Die Autoren behaupten, dass der Begriff Szene in der Sozial-, Kultur- und Wirtschaftsgeographie noch defizitär konzeptionalisiert wird, obwohl er in verschiedenen Bereichen des Alltags, der angewandten sowie der konzeptionellen Wissenschaft verstärkt breite Verwendung findet: Einerseits wird Szene umgangssprachlich zur Beschreibung und Verortung kreativer und flüchtiger Vergemeinschaftungen aber auch professioneller Netzwerke in der sogenannten Kreativ- und Wissensökonomie angewandt. Andererseits haben kultur- und sozialwissenschaftliche Disziplinen konzeptionelle Ansätze entwickelt, um mit Hilfe des Konzepts Szene eine Beschreibung der Funktionalität des Städtischen und der damit einhergehenden Prozesse der sozialen Vergemeinschaftung zu entfalten. Die Leitfrage des Beitrags lautet daher: Kann der Begriff Szene als ein kultur- und wirtschaftsgeographisches Konzept verstanden werden? Was leistet dann ein Konzept „Szene“? 